\title{
Programação em plataforma BIM e a Norma de Desempenho Brasileira: Desenvolvimento de uma aplicação para estimativa de performance acústica em projetos arquitetônicos
}

\author{
BIM platform programming and Brazilian Performance Standard: Development of an \\ application to estimate acoustic performance in architectural design
}

\author{
Juliano Lima da Silva \\ Programa de Pós-Graduação em Arquitetura e Urbanismo \\ da Faculdade Meridional - PPGARQ IMED, Brasil. \\ juliano_lima_silva@hotmail.com
}

\author{
Andréa Quadrado Mussi \\ Programa de Pós-Graduação em Arquitetura e Urbanismo \\ da Faculdade Meridional - PPGARQ IMED, Brasil. \\ andrea.mussi@imed.edu.br
}

\author{
Lauro André Ribeiro \\ Programa de Pós-Graduação em Arquitetura e Urbanismo \\ da Faculdade Meridional - PPGARQ IMED, Brasil; INESC \\ Coimbra, Portugal. \\ lauro.ribeiro@imed.edu.br \\ Thaísa Leal da Silva \\ Escolas de Ciência da Computação e Sistemas de \\ Informação da Faculdade Meridional, Brasil. \\ thaisa.silva@imed.edu.br
}

\begin{abstract}
The Brazilian Performance Standard (NBR 15.575/2013 - Housing Buildings - Performance) is changing the design process of residential buildings, imposing new acoustic performance criteria. In this Context, the present paper proposes the programming of a Revit plug-in for verifying sound insulation of walls between environments, collecting information of the building's model from a parameter database, calculating values of the elements' sound reduction index and allowing to ponder constructive systems' performance. The application aims to amplify productivity of designers and to provide greater control over technological solutions, assisting in the compliance with performance criteria.
\end{abstract}

Palavras-chave: BIM; Norma de desempenho; Isolamento acústico; Vedações verticais

\section{Introdução}

Devido ao aumento nos requisitos de qualidade de construções habitacionais, em relação à amenidade acústica de ambientes e conforto dos habitantes, uma pressão cada vez mais expressiva vêm se estabelecendo nos processos de projeto de modo a garantir o bem estar dos usuários. Neste sentido, arquitetos e engenheiros necessitam tomar decisões técnicas cruciais relacionadas à isolamento interno de ambientes para proporcionar e garantir uma performance acústica adequada em seus projetos.

Uma série de aspectos de sistemas construtivos das edificações habitacionais tornam-se indispensáveis nas decisões tecnológicas, aspectos que muitas vezes podem ser mensurados, simulados e otimizados por meio de softwares (Arjunan et al., 2014; Kim \& Kim, 2007). Por esta ótica, embora a customização de software seja uma prática comum em áreas como engenharia mecânica e design, é algo que ainda está em desenvolvimento na arquitetura, considerando as crescentes necessidades de otimização dos processos de projeto e melhorias na avaliação da performance (Matcha \& Quasten, 2009).

Este cenário se torna ainda mais evidente com o crescimento das práticas de projeto voltadas para a sustentabilidade, como a adoção de certificações ambientais por empresas no setor AEC (Arquitetura, Engenharia e Construção). Neste sentido, a necessidade de avaliar uma multiplicidade de alternativas de projeto torna-se uma prática em expansão, bem como a busca por adaptação à tecnologias como o BIM (Building Information Modeling), visando um processo de projeto voltado para edificações energeticamente eficientes e com aspectos mais sustentáveis (Jalaei \& Jrade, 2014).

Com a chegada da NBR 15.575 - Norma de Desempenho Brasileira (ABNT, 2013a), um enfoque ainda maior foi dado à performance de edificações habitacionais e com isso, diversos critérios devem ser atendidos por projetistas, fornecedores de materiais e construtores. Uma série de mudanças que altera os processos de projeto já estabelecidos na medida em que insere prerrogativas a serem atendidas e aumenta a complexidade das análises.

A Norma de Desempenho brasileira trata, dentre outras diversas exigências, do desempenho acústico de paredes de geminação entre ambientes de unidades autônomas e entre áreas privativas ou comuns nas edificações multifamiliares, sendo necessários ensaios a campo e em laboratório para verificação do desempenho em relação ao ruído aéreo de sistemas construtivos. 
Alternativas para facilitar e acelerar a predição de desempenho acústico de ambientes internos e seu atendimento à normativas tem sendo estudados com o uso de softwares de verificação e de simulação (Takahashi, 2016). Neste contexto, plug-ins e ferramentas para plataformas BIM emergiram como uma alternativa para manipular e atingir melhorias de projeto de maneira rápida nas fases iniciais de projeto (Wu \& Clayton, 2013).

Esquadrias possuem um papel considerável no isolamento acústico de paredes (Kim \& Kim, 2007), bem como os materiais que compõe camadas dos sistemas construtivos e suas especificações dimensionais. Neste sentido, este trabalho apresenta um plug-in para Revit que visa estimar o valor do índice de redução sonora $\left(R_{w}\right)$ de paredes de vedação, considerando as diferentes possibilidades dimensionais e de especificação relacionadas à portas e janelas.

O plug-in foi desenvolvido para auxiliar no atendimento à Norma de Desempenho brasileira - Edificações Habitacionais (ABNT, 2013a), contudo, pode ser customizado para se adaptar à outras normativas de abrangência internacional de órgãos como BSI (British Standards Institution) e ISO (International Organization for Standardization) (BSEN 123541, 2000; BSEN ISO 10140, 2010; BSEN ISO 717-1, 2006), devido ao fato de que a metodologia envolvida é similar e baseada no isolamento de ruído aéreo de paredes de vedação entre ambientes de uma edificação habitacional.

Ao invés de realizar cálculos com tabelas e necessitando de dados baseados em documentação fragmentada, a perspectiva de proposta no desenvolvimento desta ferramenta busca a disrupção de processos de projeto tradicionais voltados para performance, utilizando informações inerentes dos modelos BIM para coletar informações atualizadas de propriedades geométricas e de parâmetros relacionados à acústica de diferentes materiais diretamente do modelo tridimensional - uma abordagem focada na modelagem e não na documentação de projetos (Eastman, Liston, Teicholz, \& Sacks, 2008). Embora Revit não possua nativamente propriedades acústicas em suas funcionalidades, isto pode ser adicionado por meio de programação.

Contudo, a ferramenta não substitui softwares especializados de simulação, com pesquisas mais aprofundadas sendo desejáveis em casos onde é necessária uma maior precisão ou rigor técnico acerca da estimativa do índice de redução sonora em sistemas construtivos (Arjunan et al., 2014; Takahashi, 2016).

A ferramenta apresentada neste trabalho possui uma abordagem de verificação simplificada, possuindo a intenção de complementar e facilitar estimativas relacionadas ao impacto de esquadrias como portas e janelas no desempenho acústico de paredes de vedação, em uma diversidade de possíveis sistemas construtivos em projetos.

\section{Metodologia}

Uma pesquisa experimental foi conduzida de modo a programar a ferramenta. A plataforma Autodesk Revit foi escolhida como o software BIM a ser programado devido à sua comunidade de usuários já estabelecida e suas possibilidades de customização (Autodesk, 2017).

Uma das principais extensões do Revit atualmente é o Dynamo, uma extensão de VPL (Visual Programming Language) que possibilita ao usuário, através de um ambiente de programação aberto, extrair e administrar fluxos de informação paramétrica no Revit (Sanhudo, 2016). Isto é feito a partir de seus 'nodes', pacotes de funções que alteram informações de input e output, conectando informações oriundas do Revit em uma rotina de programação (Dynamo, 2017).

Através de uma rotina de trabalho criada no Dynamo (Figura 1), foi possível manipular dados extraidos de elementos BIM de um projeto para calcular o índice de redução sonora (variável $R_{w}$ ) de cada instância de parede, porta e janela da modelagem, em determinada vista, planta ou fachada no projeto da edificação.

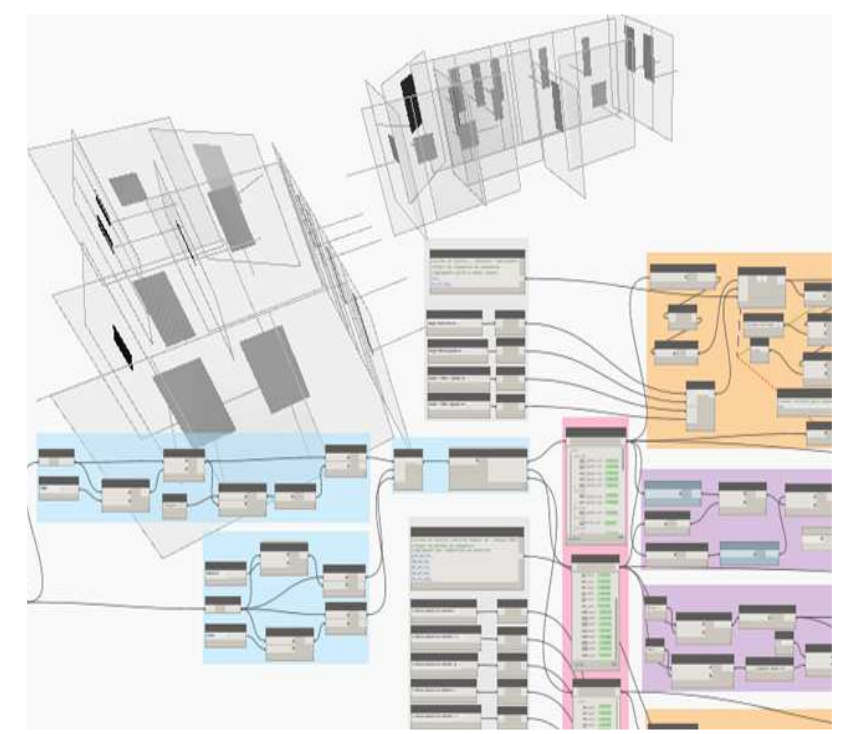

Figura 1: Programação de funções do plug-in na interface do Dynamo para Revit. 


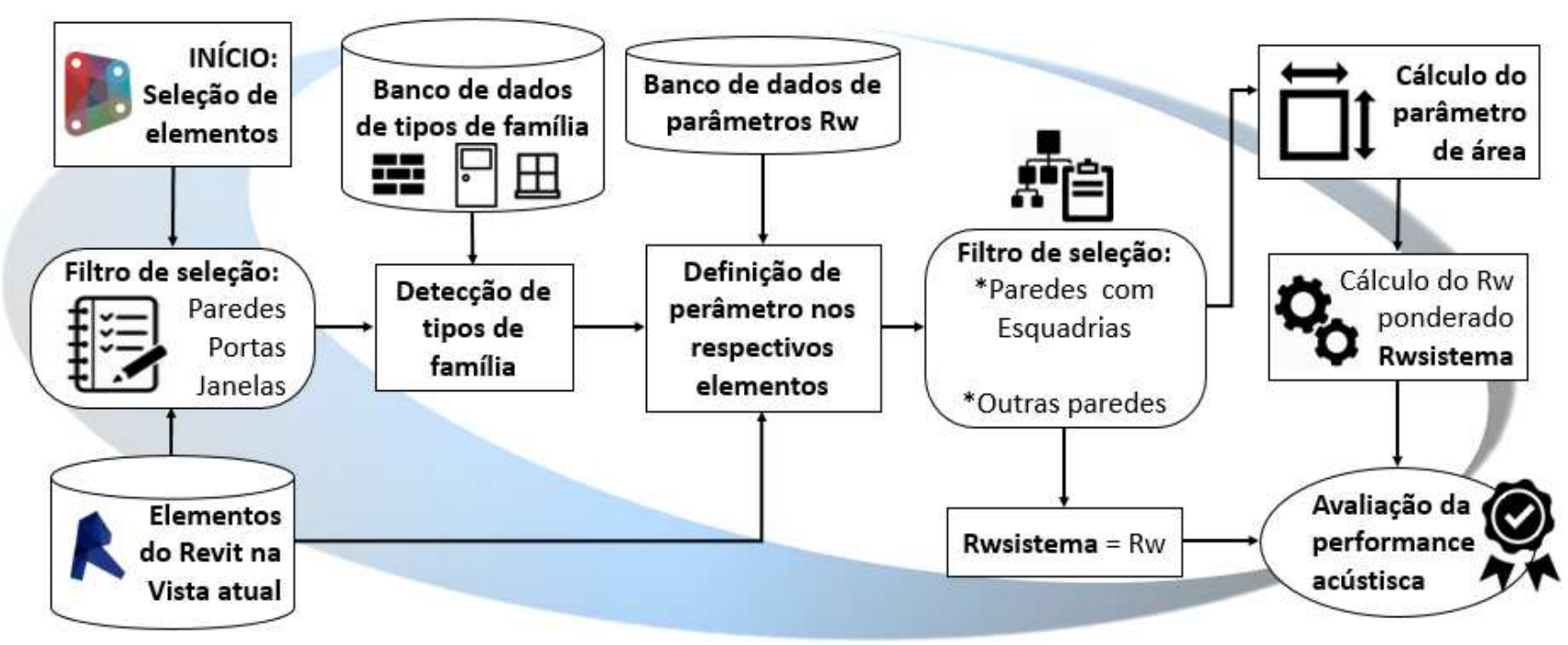

Figura 2: Funcionamento proposto para os botões da interface do plug-in.

Para manipular parâmetros de família de elementos do Revit e incluir propriedades acústicas, um arquivo de Shared Parameters foi criado, com dois principais parâmetros: $R_{w}-$ índice de redução sonora (comum a todas as paredes, janelas e portas), e Rwsistema - índice de redução sonora ponderado (presente apenas em paredes).

Então, na interface de algorítmos do Dynamo, ao executar a rotina, todos os elementos da vista atual de projeto do Revit (planta baixa, corte, fachada ou 3D) são selecionados e filtrados, apresentando todas as instâncias de parede, porta e janela na vista. A metodologia para a funcionalidade da ferramenta está apresentada na Figura 1.

Um banco de dados com listas de tipos de famílias, valores de índice de redução sonora e características de materiais são então definidos no algorítmo. Tais listas de valores então são pareadas com as instâncias de elementos do Revit selecionados na etapa anterior, os valores de parâmetros são então inseridos conforme o tipo de família correspondente em cada instância na vista de projeto. Na data deste trabalho, os parâmetros de bancos de dados seguem os valores apresentados na Norma NBR 15575-4 (ABNT, 2013b) e no Guia de Atendimento à Norma de Desempenho (CBIC, 2013), conforme Tabela 1, podendo ser expandidos para abranger outros sistemas construtivos customizados.
Tabela 1: Valores indicativos do índice de redução sonora ponderado para alguns sistemas de paredes. Adaptado de Guia Orientativo para Atendimento à ABNT NBR 15.575/2013, de Câmara Brasileira da Indústria da Construção (CBIC, 2013).

\begin{tabular}{|c|c|c|c|c|}
\hline $\begin{array}{l}\text { Tipo de } \\
\text { parede }\end{array}$ & $\begin{array}{l}\text { Largura do } \\
\text { bloco/tijolo } \\
\text { (cm) }\end{array}$ & Revestimento & $\begin{array}{l}\text { Massa } \\
\text { aprox. } \\
\left(\mathrm{kg} / \mathrm{m}^{2}\right)\end{array}$ & $\begin{array}{c}\mathrm{Rw} \\
(\mathrm{dBa})\end{array}$ \\
\hline \multirow{3}{*}{$\begin{array}{c}\text { Blocos } \\
\text { vazados } \\
\text { de } \\
\text { concreto }\end{array}$} & 9 & \multirow{3}{*}{$\begin{array}{c}\text { Argamassa } \\
1,5 \mathrm{~cm} \text { em } \\
\text { cada face }\end{array}$} & 180 & 41 \\
\hline & 11,5 & & 210 & 42 \\
\hline & 14 & & 230 & 45 \\
\hline \multirow{3}{*}{$\begin{array}{c}\text { Blocos } \\
\text { vazados } \\
\text { de } \\
\text { cerâmica }\end{array}$} & 9 & \multirow{3}{*}{$\begin{array}{l}\text { Argamassa } \\
1,5 \mathrm{~cm} \text { em } \\
\text { cada face }\end{array}$} & 120 & 38 \\
\hline & 11,5 & & 150 & 40 \\
\hline & 14 & & 180 & 42 \\
\hline \multirow{3}{*}{$\begin{array}{c}\text { Tijolos } \\
\text { maciços } \\
\text { de barro } \\
\text { cozido }\end{array}$} & 11 & \multirow{3}{*}{$\begin{array}{l}\text { Argamassa } \\
2,0 \mathrm{~cm} \text { em } \\
\text { cada face }\end{array}$} & 260 & 45 \\
\hline & 15 & & 320 & 47 \\
\hline & $11+11$ * & & 450 & 52 \\
\hline \multirow{3}{*}{$\begin{array}{c}\text { Paredes } \\
\text { maciças } \\
\text { de } \\
\text { concreto } \\
\text { armado }\end{array}$} & 5 & \multirow{3}{*}{$\begin{array}{c}\text { Sem } \\
\text { revestimento }\end{array}$} & 120 & 38 \\
\hline & 10 & & 240 & 45 \\
\hline & 12 & & 290 & 47 \\
\hline \multirow{3}{*}{ Drywall } & $\begin{array}{l}2 \text { chapas }+ \\
\text { lã de vidro }\end{array}$ & \multirow{3}{*}{$\begin{array}{c}\text { Sem } \\
\text { revestimento }\end{array}$} & 22 & 41 \\
\hline & 4 chapas & & 44 & 45 \\
\hline & $\begin{array}{l}4 \text { chapas }+ \\
\text { lã de vidro }\end{array}$ & & 46 & 49 \\
\hline
\end{tabular}

Um novo filtro é definido para detectar propriedades de paredes do tipo hospedeiro - que contenham esquadrias (janelas ou portas) anexadas, tais paredes passam por uma equação para determinar o índice de redução sonora ponderado, levando em consideração as áreas dos elementos hospedeiros para ponderar o valor de $R_{w}$ dos sistemas construtivos ( $\mathrm{R}_{\mathrm{w}}$ sistema). Outras paredes na vista (paredes 
restantes, sem janelas ou portas anexadas) têm seu valor de $\mathrm{R}_{w s i s t e m a}$ definido como o mesmo de seu $\mathrm{R}_{\mathrm{w}}$ originalmente determinado.

Por fim, os valores $R_{w}$ e $R_{w}$ sistema calculados são registrados nos parâmetros dos elementos, possibilitando aos projetistas avaliar o desempenho acústico calculado das paredes de vedação diretamente no modelo BIM. Com isso, é possível ao usuário verificar se quaisquer instâncias de parede em conjunto com portas e janelas atende aos requerimentos mínimo, intermediário ou superior da NBR 15.575.

\section{Resultados}

Os principais resultados da ferramenta desenvolvida podem ser observados por meio de aplicação prática do algorítmo durante seu uso no Revit. Embora não haja um estudo de caso específico realizado, esta seção apresenta as funcionalidades e lógica de programação envolvida. Na Figura 3, parâmetros de performance acústica ( $R_{w}$ e $R_{w}$ sistema) são inseridos em paredes, portas e janelas no Revit, por meio da customização de Shared Parameters, acompanhando os critérios metodológicos.
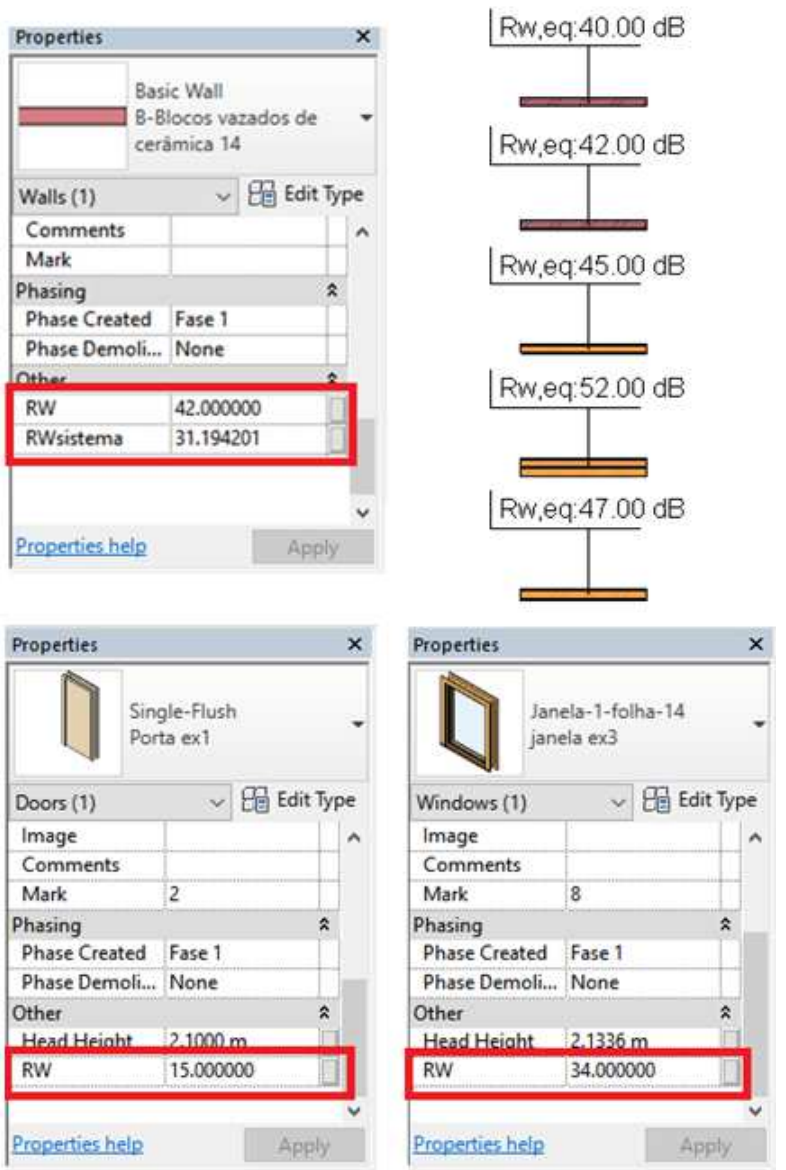

Figura 3: Definindo parâmetro Rw em paredes, portas e janelas.
O algorítmo então preenche tais parâmetros com informações de acordo com o banco de dados de índices de redução sonora cadastrado, considerando as diferentes composições de parede e esquadrias anexadas no modelo BIM, e definindo o valor de $R_{w}$ de todos os elementos correspondentes na vista atual conforme seus respectivos sistemas construtivos.

Nesta etapa, um código em Python foi implementado para facilitar a relação entre 0 banco de dados de famílias cadastrado com as múltiplas possibilidades existentes em um modelo, reduzindo a quantidade de funções do Dynamo a serem criadas para executar operações de verificação lógica entre listas e possivelmente reduzir tempos de processamento.

Durante o desenvolvimento da ferramenta, um problema relevante foi solucionado por meio de programação - por vezes o parâmetro interno de área de elementos de janelas e portas do Revit erroneamente retorna a soma de todas as áreas de superfície em sua composição. Utilizando funções do Dynamo como 'bounding box' e 'intersecção com o plano Z' conforme a orientação de cada parede, foi possível registrar a área real de janelas e portas, corrigindo o parâmetro de área necessário no cálculo de $\mathrm{R}_{\mathrm{w}}$.

Esta medida também foi necessária para cobrir uma maior quantidade de elementos de famílias a serem importadas no modelo BIM - devido ao fato de que muitas famílias são feitas por usuários e podem não possuir parâmetros específicos bem denominados como altura e largura. Isto possivelmente torna-rá o plug-in mais adaptável a diferentes particularidades de famílias de portas e janelas a serem importadas e calculadas no modelo.

A partir dos parâmetros de área de cada instância de parede, janela e porta na vista atual,o algorítmo detecta elementos de parede do tipo hospedeiro (Host Walls) e suas respectivas janelas e portas (Hosted Elements), calculando o índice de redução sonora, identificado nos parâmetros de tais elementos e no arquivo de Shared Parameters como Rwsistema.

A Figura 4 demonstra algumas possibilidades de utilizar esta ferramenta, apresentando um resultado de execução do programa em uma vista de planta baixa, com diferentes tipos de sistemas construtivos de paredes (alvenaria, concreto e drywall), com múltiplas dimensões, bem como famílias de janelas e portas em quantidades variáveis por parede - em resumo, um conjunto de possíveis situações que espelham a complexidade de projetos reais, onde a diversificação de sistemas construtivos impacta o desempenho acústico e possibilita a tomada de decisões de projeto otimizada para as soluções tecnológicas a serem empregadas. Na Figura $4, R_{w}$ e $R_{w}$ sistema de famílias são apresentados como objetos de Anotação do Revit, para melhor visualização. 


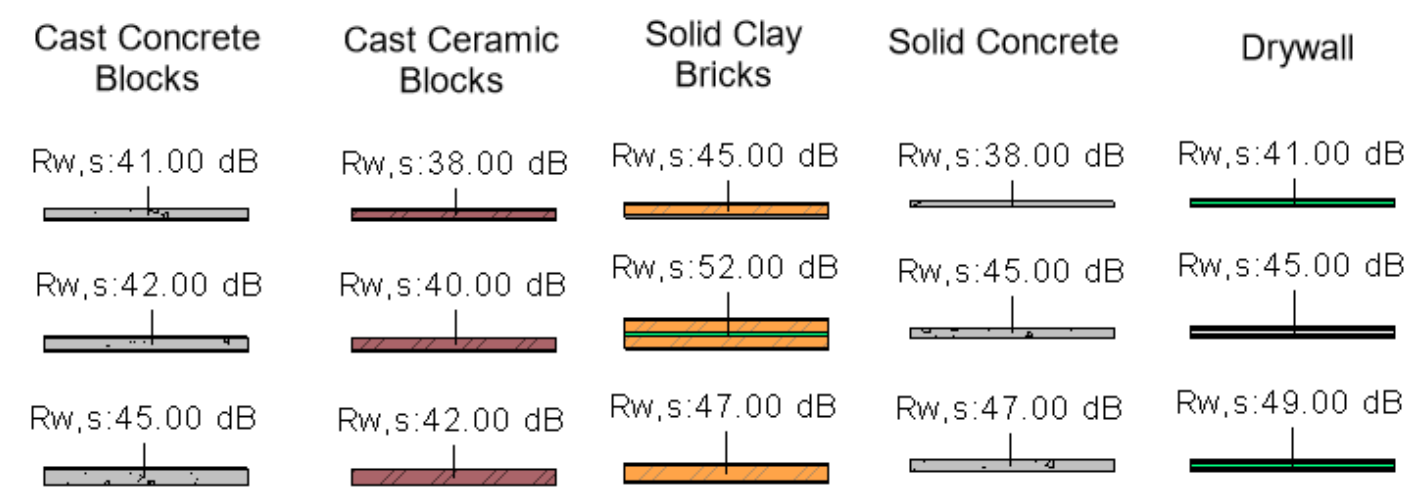

\section{Windows and Doors}
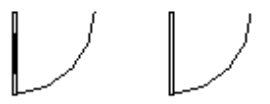

Rw: $18 \mathrm{~dB}$

Rw: $28 \mathrm{~dB}$
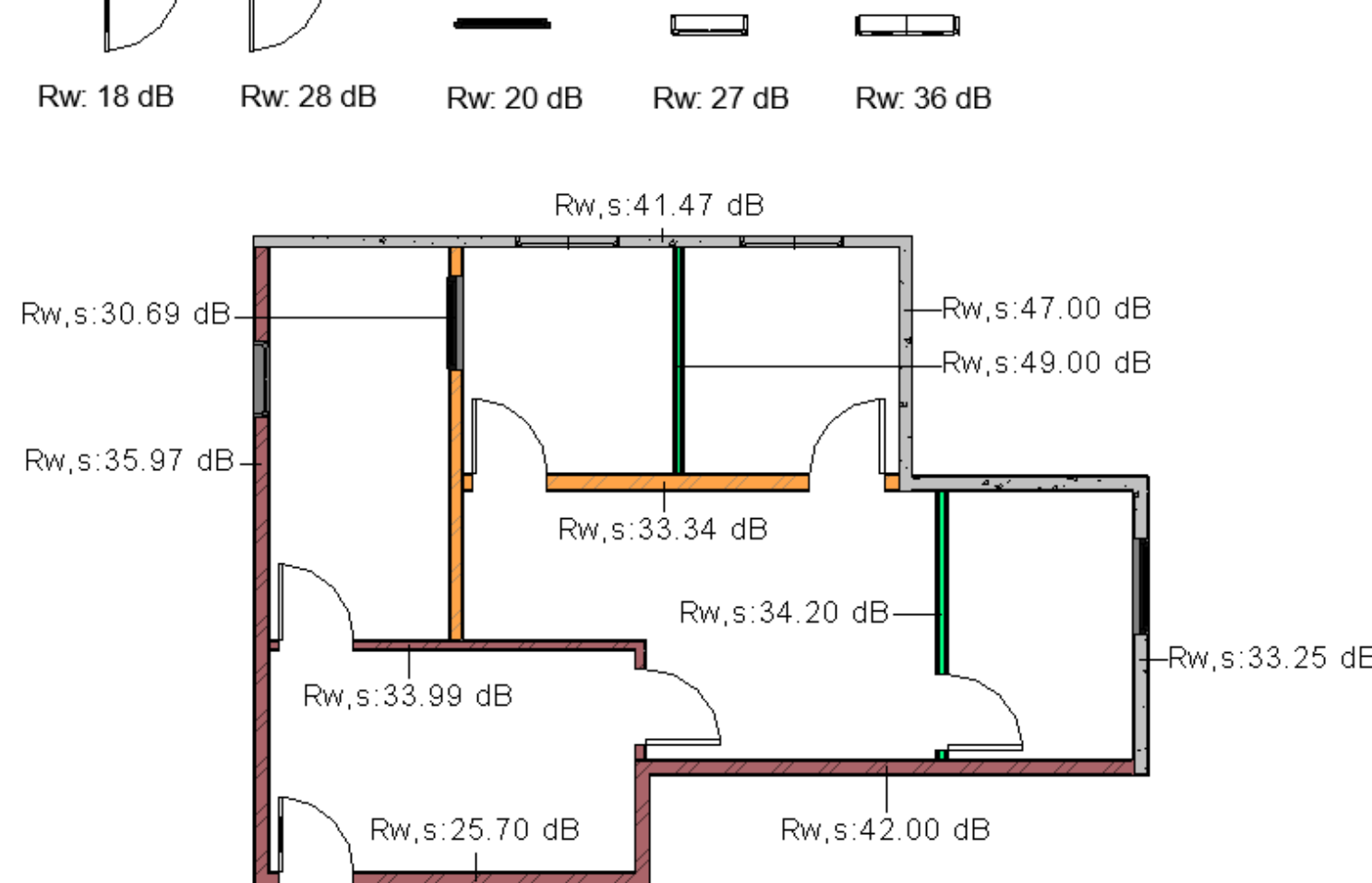

Figura 4: Estimando desempenho acústico em múltiplos sistemas de vedações com diferentes esquadrias.

\section{Conclusões}

Este trabalho apresentou o desenvolvimento e funcionalidade de um plug-in voltado para o software BIM Revit, utilizando a extensão Dynamo para estimar o desempenho acústico de paredes de divisória no contexto de edificações habitacionais e da Norma de Desempenho.

O potencial de uso do plug-in está na possibilidade de auxiliar profissionais da AEC em otimizar a performance acústica de seus projetos, facilitando a compreensão do impacto de diferentes escolhas de materiais de paredes e esquadrias durante o processo de projeto de arquitetura. $O$ plug-in portanto, é apresentado como uma alternativa aos métodos tradicionais de análise de performance, complementando outros softwares.

Para trabalhos futuros, melhorias na interface da ferramenta são planejados, com adição de botões e menus através da extensão gerenciadora de rotinas Dynamo - o software Dyno Browser. Alternativamente, uma interface programada em C\# por meio da API (Application Programming Interface) do Revit também é almejada, de modo a facilitar o uso do plug-in para o usuário final.

Melhorias no banco de dados, com manipulação de arquivos XML (eXtensible Markup Language) e interoperabilidade com planilhas do software Microsoft Excel também estão sendo feitas, de modo a organizar parâmetros de famílias do Revit e 
possibilitando customização por parte do usuário. Isto poderá trazer funções como adição, edição e remoção de certos tipos de família de listas, de acordo com as particularidades de cada projeto.

Um esforço está sendo feito para adicionar opções de verificação no plug-in, rotinas no Dynamo que verifiquem os requisitos mínimos para 0 desempenho acústico automaticamente, por meio de referenciamento aos valores Mínimo, Intermediário e Superior em diversas situações de projeto, conforme a NBR 15.575. Por exemplo, paredes entre dois dormitórios necessitam de um desempenho acústico mínimo maior do que paredes entre áreas comuns, deseja-se que o plug-in reconheça este contexto e o aplique como uma condicionante conforme cada situação na vista de projeto, alertando o projetista quanto à inconsistências durante 0 desenvolvimento do projeto arquitetônico. Tais inconsistências ou conformidades com a Norma de Desempenho podem então ser expressadas através de tabelas (schedules do Revit) e escala de cores.

Por fim, aplicação do plug-in em um estudo de caso real também é desejada, de modo a avaliar o uso do programa e como ferramenta para nos processos de projeto durante as etapas iniciais, explicitando seu potencial em auxiliar arquitetos e engenheiros em decisões tecnológicas relativas a sistemas construtivos de paredes de vedação e suas opções de esquadria.

\section{Agradecimentos}

Agradecimentos ao Programa de Pós-Graduação em Arquitetura e Urbanismo (PPGARQ) da Escola Politécnica da Faculdade Meridional (IMED), ao Programa de Suporte à PósGraduação de Instituições de Ensino Particulares (PROSUP) e à Coordenação de Aperfeiçoamento de Pessoal de Nível Superior (CAPES) pelo auxílio financeiro e bolsas que possibilitaram esta pesquisa.

\section{Referências}

ABNT. (2013a). NBR 15575-1: Edificações Habitacionais Desempenho Parte 1 : Requisitos gerais. Rio de Janeiro, Brazil: ABNT.

ABNT. (2013b). NBR 15575-4: Edificações habitacionais Desempenho Parte 4 : Sistemas de vedações verticais internas e externas - SVVIE. Rio de Janeiro, Brazil: ABNT.

Arjunan, A., Wang, C. J., Yahiaoui, K., Mynors, D. J., Morgan, T.,
Nguyen, V. B., \& English, M. (2014). Development of a 3D finite element acoustic model to predict the sound reduction index of stud based double-leaf walls. Journal of Sound and Vibration, 333(23), 6140-6155. https://doi.org/10.1016/j.jsv.2014.06.032

Autodesk (2017). Revit Store - Plugins, Add-ons and Extensions for Revit. https://apps.autodesk.com/RVT/en/Home/Index

BSEN 12354-1. (2000). Building acoustics - Estimation of acoustic performance of buildings from the performance of elements. Airborne sound insulation between rooms. BSI Standards Publication.

BSEN ISO 10140. (2010). Acoustics - Laboratory Measurement of Sound Insulation of Building Elements, Part 2: Measurement of Airborne Sound Insulation. BSI Standards Publication.

BSEN ISO 717-1. (2006). Acoustics: Rating of Sound Insulation in Buildings and of Building Elements, Part 1: Airborne Sound Insulation. BSI Standards Publication.

CBIC. (2013). Desempenho De Edificações Habitacionais: Guia Orientativo Para Atendimento à Norma ABNT NBR 15575/2013. (Gadioli Cipolla Comunicação, Ed.) (2nd ed.). Fortaleza.

Dynamo. (2017). DynamoBIM, Visual Programming for Revit. http://dynamobim.org/

Eastman, C., Liston, K., Teicholz, P., \& Sacks, R. (2008). BIM Handbook. Hoboken, New Jersey: John Wiley \& Sons, Inc.

Jalaei, F., \& Jrade, A. (2014). An Automated BIM Model to Conceptually Design, Analyze, Simulate, and Assess Sustainable Building Projects. Journal of Construction Engineering, 2014, 1-21. https://doi.org/10.1155/2014/672896

Kim, M. J., \& Kim, H. G. (2007). Field measurements of façade sound insulation in residential buildings with balcony windows. Building and Environment, 42(2), 1026-1035. https://doi.org/10.1016/j.buildenv.2005.10.036

Matcha, H., \& Quasten, G. (2009). A Parametric-Typological Tool: More Diversity for Mass Produced Single Family Homes Through Parametrized Design and Customized Mass Production. In eCAADe 27 (pp. 409-416).

Sanhudo, L. P. N. (2016). BIM for Building Sustainability Assessment: Development of a software tool for Rainwater Runoff mitigation. Faculdade de Engenharia - Universidade do Porto.

Takahashi, V. F. de M. (2016). Desempenho acústico de edificações: ferramenta computacional para avaliação. Universidade Estadual de Campinas.

Wu, C., \& Clayton, M. J. (2013). BIM-Based Acoustic Simulation Framework. In CIB W78 International Conference (pp. 99-108). Beijing, China. 